\title{
Parameter optimization of Heavy-Load Parallel Manipulator by Introducing Stiffness Distribution Evaluation Index
}

\author{
Hao Wang ${ }^{1}$, Linsong Zhang,Shunzhou Huang ${ }^{\mathrm{a}}$,Genliang Chen \\ State Key Laboratory of Mechanical System and Vibration \\ Shanghai Key Laboratory of Digital Manufacture for Thin-Walled Structures \\ School of Mechanical Engineering, Shanghai Jiao Tong University, Shanghai, 200240, P. R. China \\ ${ }^{\mathrm{a}}$ Shanghai Aerospace Equipment Manufacturer, Shanghai, 200245, China
}

\begin{abstract}
Aiming at the heavy-load parallel robotic manipulators, other than classic sequential 'topology-dimension-structure' parameter design methodology, this paper proposes a new way to optimize dimension and structure parameters ${ }^{2}$ simultaneously. To deal with the low-speed but heavy load working environment, a new type of stiffness performance evaluation index is introduced based on kineto-elastic statics (KES) analysis. This stiffness distribution index, mainly focuses on the stiffness distribution among all parts in the manipulator, along with two other dynamic performance indices and some dimensional constraints, formulate a multi-objective optimization model.Then the particle swarm optimization (PSO) algorithm is introduced to search for the optimum parameters. This method is applied on a widely used TRICEPT parallel manipulator, the dynamic performances before and after optimization are compared to verify this effectiveness of this method.
\end{abstract}

Keywords: Parameter optimization; Stiffness distribution index; KES analysis; PSO algorithm; Parallel manipulator.

\section{Introduction}

Different from normal industrial robots, the design optimization objective for heavy-load parallel robot manipulators includes not only the dimension-related kinematics performances, but also the structure-related dynamics performances, such as the carrying capacity, stiffness, inertia, in the design process. Therefore, the design variables should involve both dimensional and structural parameters.

In most existing researches, dimensional synthesis methods deals with the kinematic performance[1, 2] optimization, such as workspace[1, 3] maximization, condition number[4-6] of the Jacobian matrix for evaluating the accuracy of a robot; Dexterity[7-9] can also be served as a measure to assess kinematic performances; Manipulability[10] index to describe the ability of the end-effector to move in all directions; Motion/force transmissibility[11] and transmission angle[12] indices, etc. Meanwhile, some researchers have also paid their attention to the dynamic performance optimization, such as stiffness[13, 14], precision[15, 16], actuation energy consumption[17, 18], dynamic isotropy[18], and inertial and centrifugal/Coriolis effects[19, 20]. As it shows, the aforementioned researches mainly concentrate on separate kinematic or dynamic performances, and even in dynamic performance optimization, design variables in these studies are also dimensional parameters, seldom structural parameters are involved.

To overcome this situation, some scholars tried to use multi-objective optimization[8, 21-27] method

${ }^{1}$ Corresponding author. Tel.: +86 213420 6786; fax: +86 2134204542.

E-mail addresses: wanghao@sjtu.edu.cn, (Hao Wang)

${ }^{2}$ Just to be clear, in most cases, dimensional parameters normally indicate length of linkages, and structural parameters involves sectional area parameter of both linkage and moving platform. 
to obtain the best performance including both kinematics and dynamics. Among them, Huang[22] proposed a new methodology for the integrated design of a 4-DOF SCARA pick-and-place robot, whose objectives include lightweight, elastic dynamic behavior, and motor size; To optimize stiffness, manipulability, condition number, dynamic mass property of a linear Delta robot, Kelaiaia[23] formulated another parameter design model; Bounab[24] presented a dimensional synthesis of the Delta parallel robot, focusing on workspace as well as stiffness and dexterity; Merlet et al.[26] have successfully used the interval analysis for the design of parallel kinematic machines (PKM).

However, none of the above mentioned methods deal with the parameters design for heavy-load working condition. Indeed, some researches[22, 23, 28] have also acquired both dimension and structure parameters after optimizations, but their targets are high-speed pick-and-place robot, bearing little or no external load; Besides, the stiffness performances involved in these objectives almost all refer to end-effector stiffness, none have discussed the stiffness distribution of all elastic parts in the whole manipulator, which should be a key factor in heavy-load manipulator design. Therefore, new evaluation indices should be proposed to fill the void.

Since the stiffness (or compliance) of a robot manipulator reflected at its end-effectorhas great influence to successfully conduct contact or non-contact tasks. As a matter of fact, the stiffness of robot manipulators usually represents the accuracy for satisfying the desired position and/or force requirements [29]. Generally, the stiffness analysis explains the effect of the applied forces and/or torques on the deformation displacements of the end-effector, which is defined as the stiffness matrix $\mathbf{K}$. Also from the mechanics theory, $\mathbf{K}$ is a $6 \times 6$ semi-definite non-negative matrix, in which the non-diagonal parts of this matrix represent the coupling between the translational and rotational deformations[30]. Besides, since the stiffness matrix $\mathbf{K}$ is configuration-dependent, which means it varies in the whole workspace, the corresponding analysis is known as the "stiffness maps", which describe the end-effector stiffness as a function of the manipulator configuration [31, 32].

Speaking of the end-effector stiffness "evaluation", many indices have been proposed. As mentioned above, the stiffness analysis always corresponds to a stiffness matrix $\mathrm{K}$, which is often required to be transformed into a stiffness index to assess the stiffness performance of a parallel manipulator. Generally speaking, a local stiffness index can be directly correlated with the end-effector stiffness matrix by means of different mathematical operators. Feasible options include determinant, trace, eigenvalues, norm at a given posture, etc., as proposed in Refs.[1, 30, 31, 33-41]; in the meantime, some kinematic performances, including the dexterity[7-9] can also be served as an indirect measure of stiffness.

Specifically, since the maximum and minimum eigenvalues of a stiffness matrix $\mathrm{K}$ represent the most and least rigidity values in the corresponding eigenvectors directions, they can be chosen as the stiffness indices[1, 37, 38] to define upper and lower bounds of the end-effector stiffness; the determinant of the stiffness matrix[37] is invariant in similarity transformation, which gives this index a fantastic feature of reference-frame independent; the condition number is normally adopted as a dexterity index, so the ref.[42] has combined it with the stiffness evaluation index; the trace is another good way to measure the deformation displacements per unit of external wrench, however, since different components of the stiffness matrix have different dimensions, the sum does not have a full physical meaning; the norm of a matrix is similar to what an absolute value is for a real number, so the norm of the stiffness matrix[43] provides an assessment on how much the stiffness matrix differs from zero. Also, the strain energy method[44] was adopted to get the stiffness property of the end-effector.

A local index of stiffness performance is inaccurate for design analysis, as well as incomprehensive 
for a comparison among different designs. Therefore, the global index was proposed to evaluate the stiffness performance in the whole workspace. Graphical method[1, 34, 42] is one way that are based on plotting curves connecting configurations having the same local stiffness index; other global indices provided in Ref.[39] can be used to maximize the stiffness along some specific direction(s), or to detect and avoid a design with weak stiffness performance along a specific direction, or to find a isotropic property in form of stiffness performance, etc.

However, still, almost all of the above indices focused on the end-effector stiffness performance, seldom had paid attention to how the stiffness of each linkage or joint distributed in the whole mechanism. Therefore, for such manipulators working under specific conditions, some new stiffness evaluation indices appeared to be vital for assessing the contribution of each elastic part to the stiffness ofthe whole system. Based on these stiffness distribution indices, the design and optimization for the manipulator structurecan be conducted, then such as the vulnerable part(s) of the manipulator can be found and strengthened.

In this paper, for the specific requirement of heavy-load environment, the idea of acquiring both kinematic and structural optimum parameters simultaneously in the conceptual design process is proposed: after certain simplification assumptions are made, both kinds of parameters will be treated as the design parameters; a new stiffness distribution density index is introducedin the optimization objectives along with others; then, after the optimization modelbeing constructed and solved, the analytical solutions can be achieved using particle swarm optimization (PSO) algorithm.

The remainder of the paper is organized as follows: the definition and construction of stiffness distribution index based on elastic potential energy density is discussed in Section 2; Section 3 provides a unified systematic procedure to construct this dimension \& structure optimization model, which contains the choice of variables, definition of performance indices and constraints; in Section 4, a brief introduction of our optimization algorithm PSO is provided; in Section 5 a widely applied industry robot TRICEPT is presented to show the effectiveness and advantage our optimization method. A brief conclusion and open discussion will be given in Section 6 .

\section{Stiffness distribution density index based on elastic potential energy}

In a low-speed but heavy-load working environment, such as flexible forming process, not only the end-effector stiffness[45, 46] should be considered, the most fragile part in the manipulator should also be paid special attention according to the 'bucket effect'. To deal with this situation, a stiffness distribution index should be proposed. However, just as it was mentioned in introduction, stiffness matrices can't be used to evaluate their distribution directly, for the dimensions of stiffness matrices could be quite different among different parts. However, from the view of elastic mechanics,the stiffness matrix is identical to the Hessian obtained from the second differentials of the elastic potential energy function[47], the stiffness of a structure can be measured by the elastic potential energy density (energy per unit volume).

By introducing stiffness distribution density (SDD) index, contribution of elasticity of each part or sub-structure to the whole manipulator is defined; Meanwhile, since the index is defined in the light of energy analysis, it endows merits such as dimensionless and invariant under coordinate transformation. Since most parallel manipulators have no large deformation in manipulation, it is assumed that the rigid-body displacements do not couple with the elastic deformation in calculation. This assumption also ensures that elastic potential energy of different parts can be calculated independently. According to relevant dynamics theory, the kineto-elastic statics (KES) model of a manipulator can be written as:

$$
\mathbf{K} \boldsymbol{U}^{e}=\mathbf{F}-\mathbf{M} \mathscr{G}^{\mathbf{Q}}
$$


where $\boldsymbol{U}^{e}$ represents elastic deformation in the pre-assigned generalized coordinate, $\boldsymbol{U}^{\mathrm{r}}$ is the rigid body displacement, $\mathbf{M}$ denotes the overall mass matrix of the system, $\mathbf{K}$ is the overall stiffness matrix, $\boldsymbol{F}$ means the generalized external force acting on the manipulator. Damping is ignored in this paper.

From Eq.(1), elastic deformation vector of the manipulator $\boldsymbol{U}^{e}$ is relevant to external force. Assuming that $\varphi_{S}=U^{e}$ is the elastic deformation vector of the manipulator in KES analysis, the overall elastic potential energy density of the manipulator is formulated as:

$$
\rho^{S}=\frac{\frac{1}{2} \boldsymbol{\varphi}_{S}^{T} \mathbf{K} \boldsymbol{\varphi}_{S}}{\sum_{i=1}^{n} V_{i}}
$$

where $V_{i}$ denotes the volume of each linkage.

Similarly, the elastic potential energy of the $i$-th elastic part can also be acquired:

$$
\rho_{i}^{S}=\frac{\frac{1}{2} \boldsymbol{\varphi}_{S i}^{T} \mathbf{K}_{i} \boldsymbol{\varphi}_{S i}}{V_{i}}
$$

where $\mathbf{K}_{i}, \boldsymbol{\varphi}_{S i}$ are stiffness and elastic deformation vector of the $i$-th elastic part.

As can be seen in Eq.(2) and (3), $\rho^{S}$ and $\rho_{i}^{S}$ represent 'overall' and 'individual' elastic potential energy density respectively. Thus, the stiffness density distribution of the $i$-th elastic part engaged in the whole manipulator can be easily calculated

$$
\gamma_{i}^{\rho}=\frac{\rho_{i}^{S}-\rho^{S}}{\rho^{S}}
$$

If the value $\gamma_{i}^{\rho}$ is positive, it means the elastic potential energy density of this linkage is above average, the stiffness of this part should be strengthened. Negative value means elastic potential energy density of this part is below average.

The above analyses are based on the instantaneous structure hypothesis, which considers the manipulator as a structure in specific configuration, so calculations are all local. In order to obtain the stiffness performance during the entire path, an integration is required

$$
\mathrm{g}_{\rho i}=\frac{\int_{L} \rho_{i}^{s} d s}{\int_{L} d s}
$$

where $L$ is the machining path in actual forming process or other low-speed but heavy-load situations.

Then the SDD index can be defined:

$$
\varphi_{\mathrm{SDD}}=\frac{g_{\rho i}{ }^{\max }-g_{\rho i}{ }^{\min }}{g_{\rho i}^{\max }}
$$

This index indicates the uniformity of stiffness distribution of each linkage in the manipulator during the whole machining path (or workspace depending on specific requirement), smaller value of this index $\varphi_{\text {SDD }}$ shows better evenness of stiffness distribution in the manipulator.

\section{Parameter optimization design process}

In order to obtain both the dimensional and structural parameters in the optimization process, structure and shape of the parts are simplified to a permissible level: the linkages are treated as a standard beam element, and the moving platform is assumed to have a regular shape. Considering the 
optimization is conducted in the conceptual design stage, these simplifications are acceptable, specific refinement of each individual part can be carried out after this process.

\subsection{Optimization design variables}

Design variables can be extracted from the general structure of a parallel manipulator, as shown in Fig.1, in most cases linkages and the moving platform are the optimization design targets. During the design process, after simplification hypothesis being established, linkage can be treated as a standard spatial beam element or an assembly of such elements, as shown in Fig. 2(a), its design variables normally includes length $l$ and its sectional area $A$; Meanwhile, the moving platform is usually simplified into a regular cross-section geometry, such as a circle or square, then its design variables involves cross-sectional area $A$ and its thickness $h$, as shown in Fig.2(b).

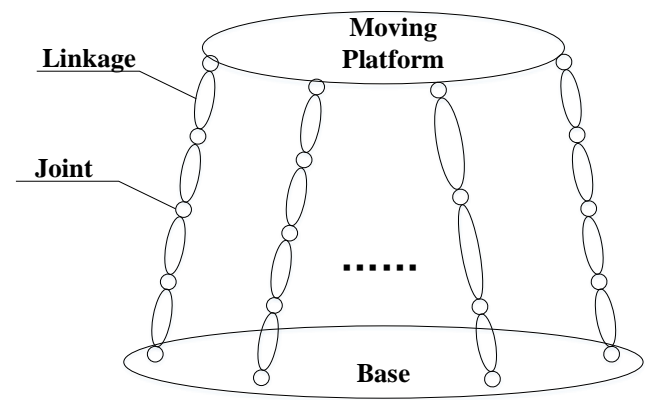

Figure 1. General structure of a parallel manipulator

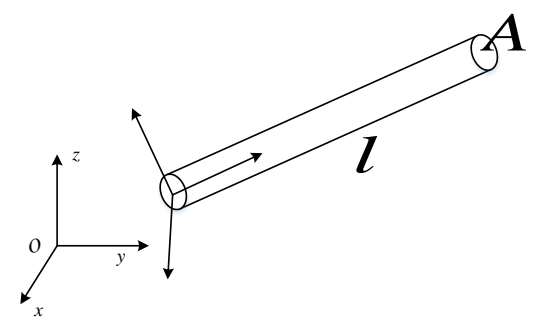

a) linkage parameters

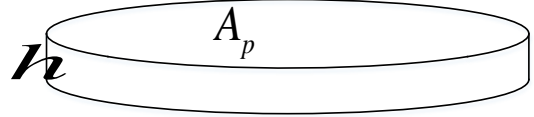

b) moving platform parameters

Figure. 2 Optimization design variables

The optimization objectives of this study mainly considers the dynamic performances, including the stiffness (or deformation) and mass property. Referring to the elastic mechanics[48], both of the standard stiffness matrix and mass matrix can be acquired, in the form of $6 \times 6$ symmetric positive definite matrices, which are all related to the design variables $(l, h, A)$ mentioned above.

Therefore, to get a better dynamic performance, not only the dimensional parameter (embodied by linkage length), but also the structure parameters (such as cross-sectional area and thickness) should also be involved in the optimization process. In summary, these design variables include

(1) Dimensional parameters: $X=\left[\begin{array}{llll}l_{1} & l_{2} & \cdots & l_{n}\end{array}\right]$, with $l_{i}$ the length of each linkages, $n$ is the number of linkages;

(2) Structural parameters: $Y=\left[\begin{array}{llllll}A_{1} & A_{2} & \cdots & A_{n} & A_{p} & h_{p}\end{array}\right]$, with $A_{i}$ and $A_{p}$ being the cross-sectional area of linkage and the platform, $h_{p}$ means the thickness of the moving platform;

\subsection{Optimization Performance Evaluation Indices}

In this paper, different from other researches, the actual machining environmentis considered, not just the natural vibration analyses[13, 15, 49, 50].Sincethe flexible forming process is mainly concerned in our study, which is a low-speed but heavy-load situation, 4 dynamic performances are 
introduced, including (1) a stiffness distribution density (SDD) index which considers the uniformity of stiffness distribution in the manipulator during the whole machining path; (2) The end-effector stiffness indices, which involve a stiffness model reduction method, will consider two practical requirement, including average and minimum end-effector stiffness; (3) a minimum mass index which satisfies the minimum weight requirement in conceptual design process.

\subsubsection{Stiffness distribution density (SDD) index $\varphi_{\mathrm{SDD}}$}

Since this performance index is well illustrated in Section 2, it will be simply listed here for clarity. According to Section 2, this index is defined by

$$
\varphi_{\mathrm{SDD}}=\frac{g_{\rho i}{ }^{\max }-g_{\rho i}{ }^{\min }}{g_{\rho i}{ }^{\max }}
$$

This index indicates the uniformity of stiffness distribution of each linkage in the manipulator during the whole machining path (or workspace depending on specific requirement). By introducing this index, contribution of elasticity of each part or sub-structure to the whole manipulator is defined; Meanwhile, since the index is defined in the light of energy analysis, it endows merits such as dimensionless and invariant under coordinate transformation.

\subsubsection{End-effector stiffness index $\varphi_{\mathrm{SEE}}$}

Based on the KES analysis, the stiffness matrix of the whole manipulator can be obtained, as the matrix $\mathbf{K}$ in Eq.(1). Since it is known to all that this $n \times n$ stiffness matrix is defined on the generalized coordinates, which involves every elastic part in the manipulator; In order to obtain the stiffness of the end-effector, certain reduction technique will be adopted based on Guyan's static condensation method[51]. From the statics Eq.(1), generalized coordinates $\boldsymbol{U}^{e}$ will be divided into the preserved part $\boldsymbol{U}^{p}$ and the reduced part $\boldsymbol{U}^{c}$, then the equation can be reformed into:

$$
\left(\begin{array}{ll}
\mathbf{K}_{\mathrm{cc}} & \mathbf{K}_{\mathrm{cp}} \\
\mathbf{K}_{\mathrm{cp}}^{\mathrm{T}} & \mathbf{K}_{\mathrm{pp}}
\end{array}\right)\left(\begin{array}{l}
\boldsymbol{U}^{c} \\
\boldsymbol{U}^{p}
\end{array}\right)=\left(\begin{array}{l}
\boldsymbol{F}^{c} \\
\boldsymbol{F}^{p}
\end{array}\right)
$$

Based on the Guyan's hypothesis, the forces on the reduced coordinates is assumed to be zero, which is $\boldsymbol{F}^{\mathrm{c}}=\boldsymbol{O}$, then $\boldsymbol{U}_{\mathrm{c}}=-\mathbf{K}_{\mathrm{cc}}^{-1} \mathbf{K}_{\mathrm{cp}} \boldsymbol{U}_{\mathrm{p}}$, and

$$
\boldsymbol{U}^{\mathrm{e}}=\left(\begin{array}{c}
\boldsymbol{U}^{\mathrm{c}} \\
\boldsymbol{U}^{\mathrm{p}}
\end{array}\right)=\left(\begin{array}{c}
-\mathbf{K}_{\mathrm{cc}}^{-1} \mathbf{K}_{\mathrm{cp}} \\
\mathbf{I}_{(3 \times 3)}
\end{array}\right) \boldsymbol{U}^{\mathrm{p}}=\overline{\mathbf{T}} \boldsymbol{U}^{\mathrm{p}}
$$

According to the energy equivalence principle, the deformation energy before and after the reduction should be the same,

$$
\frac{1}{2} \boldsymbol{U}^{e T} \mathbf{K} \boldsymbol{U}^{e}=\frac{1}{2} \boldsymbol{U}^{p T} \mathbf{K}_{\mathrm{End}} \boldsymbol{U}^{p}
$$

Then the end-effector stiffness can be derived accordingly

$$
\mathbf{K}_{\text {end }}=\overline{\mathbf{T}}^{\mathrm{T}} \mathbf{K} \overline{\mathbf{T}}=\mathbf{K}_{\mathrm{pp}}-\mathbf{K}_{\mathrm{cp}}^{\mathrm{T}} \mathbf{K}_{\mathrm{cc}}^{\mathrm{T}} \mathbf{K}_{\mathrm{cp}}
$$

This reduction technique is a simplified version of other sophisticated Cartesian stiffness calculation method[52]. However, in this study, to keep the uniformity of the KES analysis method, the reduction method is adopted; besides, accurateness is good enough according to our design requirement, comparing to others.

Since the calculated stiffness matrix $\mathbf{K}_{\text {end }}$ is also configuration dependent, an index for this specific 
configuration $k_{e}$ is defined

$$
k_{e}=\operatorname{tr}\left(\mathbf{K}_{\text {end }}\right)
$$

1) In order to indicate the average stiffness performance of the end-effector along a machining path. The corresponding stiffness performance index is defined

$$
\varphi_{\mathrm{SEE}}^{a}=\frac{\int_{L} k_{e} d s}{\int_{L} d s}
$$

Obviously, greater value of this index reflects better end-effector stiffness performance.

2) Meanwhile, the worst stiffness scenario should be avoided in most of the application cases, therefore, other than the 'average stiffness performance' mentioned in Eq.(12), the minimum stiffness value along the working path is identified by adopting another stiffness performance index

$$
\varphi_{\mathrm{SEE}}^{m}=\left.\min k_{e}\right|_{L}
$$

It can be understood that larger value of this index means better stiffness performance, which can better avoid the worst stiffness case along the practical working path.

\subsubsection{Manipulator Mass index}

In the design process of an industrial manipulator, economic issue is a major concern, therefore, material cost saving is normally involved in the manipulator design process, mainly reflects in the total mass of the moving parts. Therefore, a mass index is defined:

$$
\varphi_{M}=\sum M_{i}
$$

where $M_{i}$ is the mass of the $i$-th part in the manipulator, normally including linkage and the moving platform.

\subsection{Optimal objective function}

Based on the above mentioned dynamic performance indices, the corresponding optimal objective functions can also be defined, reflecting its dynamic performances.

Since the goal of optimal objectives $\varphi_{\mathrm{SDD}}$ and $\varphi_{M}$ is to find the minimum value, while the target of $\varphi_{\mathrm{SEE}}$ is to get the maximum, so an inversion of $\varphi_{\mathrm{SEE}}$ is conducted. Therefore, a weighted optimal objective function can be formulated:

$$
\min \quad F=-\omega_{1} \varphi_{\mathrm{SDD}}+\omega_{2} \frac{1}{\varphi_{\mathrm{SEE}}^{a}}+\omega_{3} \frac{1}{\varphi_{\mathrm{SEE}}^{m}}+\omega_{4} \varphi_{M}
$$

where $\omega_{i}$ is the weighted coefficient, bigger value means more importance of this constraint, considering the specific design requirement. In this paper, all objectives are equally important to, so their weighted coefficient are set to be the same.

\subsection{Constraints}

During the optimization process, a huge amount of dimension-structure parameters can be solved, but not all of them can be adopted, therefore, the constraints are included to narrow the search field. In this paper, the following constraints are considered.

(1) Assembly capability: the dimensions of each part should be able to assembled together, avoiding the situations with linkage too short to assemble together or too thick to collide.

(2) Length ratio constraint: the ratio of length between two linkages should be within the reasonable range. Aside from the artistic reasons, too much of the difference between two linkages may 
easily result in a singularity situation, and/or a workspace insufficiency problem due to the translational displacement restriction.

(3) Sectional area ratio constraint: the ratio of sectional area of two linkages, or the sectional area between moving platform and fixed platform should be within the reasonable range.

Based on the penalized function, the constraints of the optimization problem can be assembled

$$
f_{\text {constraint }}=\sum_{i=1}^{n} w_{i} C_{i}\left(\boldsymbol{X}_{d}, \boldsymbol{X}_{s}\right)
$$

where $n$ denotes the number of constraints, $C_{i}\left(\boldsymbol{X}_{d}, \boldsymbol{X}_{s}\right)$ indicates the $i$-th constraint, $C_{i}=1$ means the design variables are not satisfied in the constraints, $C_{i}=0$ means the opposite; $w_{i}$ is the weighted coefficient.

\subsection{Optimization model construction}

Considering all the above analysis of objectives and constrains, a universal optimization model can be formulated:

$$
\left\{\begin{array}{l}
\min \quad F=-\omega_{1} \varphi_{\mathrm{SDD}}\left(\boldsymbol{X}_{d}, \boldsymbol{X}_{s}\right)+\omega_{2} \frac{1}{\varphi_{\mathrm{SEE}}^{a}\left(\boldsymbol{X}_{d}, \boldsymbol{X}_{s}\right)}+\omega_{3} \frac{1}{\varphi_{\mathrm{SEE}}^{m}\left(\boldsymbol{X}_{d}, \boldsymbol{X}_{s}\right)}+\omega_{4} \varphi_{M}\left(\boldsymbol{X}_{d}, \boldsymbol{X}_{s}\right) \\
\text { subject to }: f_{\text {conssraint }}=\sum_{i=1}^{n} w_{i} C_{i}\left(\boldsymbol{X}_{d}, \boldsymbol{X}_{s}\right)=0
\end{array}\right.
$$

where $\boldsymbol{X}_{d}=\left[l_{1}, l_{2}, \cdots l_{n}\right]$ denotes the dimensional parameters, which in most cases meaning length of linkages , $\boldsymbol{X}_{s}=\left[A_{1}, A_{2}, \cdots A_{n}, A_{p}\right]$ means the structural parameters, which normally involving sectional area parameter of both linkage and moving platform。

\subsection{Optimization algorithm}

With regard tothe multi-parameter mechanical optimization problem, considering thecomplicated boundary condition and constraints, normal optimization methods (such as gradient-based algorithm) are not capable of dealing with such problems. As the matter of fact, traditional optimization methods normally deal with constraint-free and differentiable problems, it will not be suitable when the problem is beyond these conditions.Specifically, in our case, the parameter optimization is a non-explicit, highly-nonlinear, multi-constraint problem, therefore,evolutionary algorithms (EA) are introduced, such as genetic algorithm (GA) and particle swarm optimization (PSO) algorithm. More specifically, aiming at the multi-objective optimization, a weighted sum optimization offers a simple but effective choice; also the Pareto-optimal solutions[23, 24]can also provide a set of non-dominated solutions, so additional analysis is needed for the choice of optimal solution.

In this paper, to deal with the multi-objective optimization problem, the simple weighted sum method is adopted, as shown in Eq.(17), its principle is to apply a weighting coefficient to each objective function and then to take the sum of a weighted objective functions. Among different evolutionary algorithms, the PSO algorithm[53]does not use the step 'selection', and all population survive from the beginning of a trial to the end. The interactions result in iterative improvement of the quality of problem solution over time, which has been proved its efficiency in different application domains. Therefore, it is chosen as our optimization algorithm.

The most standard type of PSO implementation defines the particles' behaviors in two formulations. The first formula Eq.(18) is adopted to update the velocity or step size of the particle, and the 2nd one Eq.(19) moves the particle by adding the evolved velocity to its previous position.

$$
v_{i d}^{k+1}=v_{i d}^{k}+c_{1} r_{1}\left(P_{i d}-z_{i d}^{k}\right)+c_{2} r_{2}\left(P_{g d}-z_{i d}^{k}\right)
$$




$$
z_{i d}^{k+1}=z_{i d}^{k}+v_{i d}^{k+1}
$$

where $i=1,2, \ldots, N, d=1,2, \ldots, D$; and $\mathrm{N}$ denotes the number of particles, D means the dimension of each particle, $\mathrm{k}$ shows the iteration number. Besides, $c_{1}$ and $c_{2}$ are the weighted factor, $r_{1}$ and $r_{2}$ are the reviewer mentioned random number between the range $[0,1]$.

In order to improve the convergence performance of the standard PSO algorithm, some evolved methods $[54,55]$ are also proposed, since the optimization method are not the focal point of this paper, further dwelling will not be introduced.

\section{Case study: application to the TRICEPT parallel robot}

A TRICEPT manipulator is introduced to illustrate our proposed optimization method, where it works as a flexible forming machine undertaking heavy load from the work piece. As shown inFig.3(a), the main body of the TRICEPT parallel manipulator is a 3-DOF parallel mechanism, consisting of a moving platform linked to the base by three identical UPS limbs (the actuated limbs) and one UP limb (the constrained passive limb). The platform is rigidly fixed to one extremity of the UP limb. Here, U, P, and $\mathrm{S}$ represent universal, prismatic, and spherical joint, respectively, and underlined $\underline{\mathrm{P}}$ denotes an actuated prismatic joint driven by a servomotor.

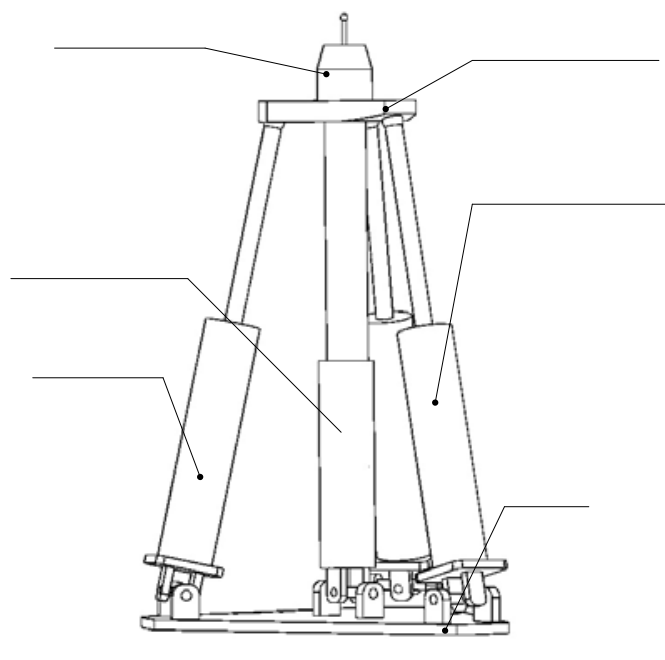

(a) Structure of the manipulator

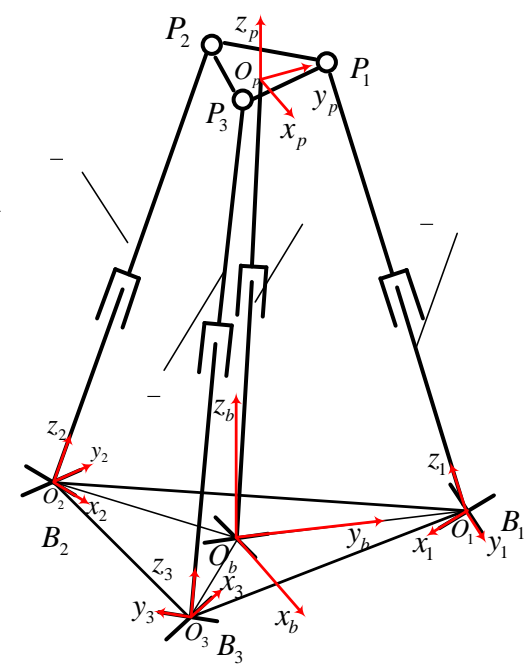

(b) Scheme of the manipulator

Figure3. A TRICEPT manipulator

Fig. 3(b) shows the scheme of the TRICEPT. The joints locating on the base and the platform are lying on circles with radii $r_{b}$ and $r_{p}$, respectively. The reference coordinate system $O_{b}-x_{b} y_{b} z_{b}$ is placed with $O_{b}$ being located at the center of the U joint of the UP limb (Constrained Limb), the $y_{b}$ axis being the axis pointing from $O_{b}$ to $B_{1}$, and $z_{b}$ axis being vertically placed, $x_{b}$ axis following the right-hand rule. The body-fixed coordinate system $O_{p}-x_{p} y_{p} z_{p}$ on the moving platform is placed in the plane where $O_{p}$ is located with the $z_{p}$ axis being the $O_{b} O_{p}$ axis of the UP limb and $y_{p}$ axis being the direction pointing from $O_{p}$ to $P_{1}$. Meanwhile, the reference and body-fixed systems $O_{i}-x_{i} y_{i} z_{i}$ associated with $\operatorname{limb} i(i=1,2,3)$ are placed in the center of each universal joint, with $z_{i}$ axis pointing from $B_{i}$ to $P_{i}$, and the $x_{i}$ and $y_{i}$ axis being the same direction of two shafts of the universal joint, with the $y_{i}$ axis being tangent to the circumcircle. 
The original geometric parameters before optimization is listed in Table 1, which exists as a comparison model. In this model, material is assumed to steel for all parts.

\begin{tabular}{cc} 
Table 1. Structural parameters of A TRICEPT manipulator & Parameters \\
\hline Part Name & circumcircle radius $r_{\mathrm{p}}=0.1 \mathrm{~m}$ \\
Moving Platform & circumcircle radius $r_{\mathrm{b}}=0.225 \mathrm{~m}$ \\
Bower Link in Actuated Limbs & diameter $d=0.05 \mathrm{~m}$; length $l_{1}=0.4 \mathrm{~m}$ \\
Upper Link in Actuated Limbs & diameter $d=0.025 \mathrm{~m}$; length $l_{2}=\left(\overline{B_{i} P_{i}}-0.4\right) \mathrm{m}$ \\
Lower Link in Constrained Limb & diameter $d=0.075 \mathrm{~m}$; length $l_{1}=0.45 \mathrm{~m}$ \\
Upper Link in Constrained Limb & diameter $d=0.05 \mathrm{~m}$; length $l_{2}=\left(\overline{O_{b} O_{p}}-0.45\right) \mathrm{m}$
\end{tabular}

Elastic modulus E=210e9MPa; Shear modulus G=79e9MPa; Density $\rho=7800 \mathrm{~kg} / \mathrm{m}^{3}$

Since TRICEPT parallel manipulator is studied comprehensively, its kinematics and rigid-body dynamics analyses[56, 57] are omitted in this paper, only its elastic dynamics analysis, is briefly introduced for convenience.

\subsection{KEDmodel of a TRICEPT manipulator}

In elastic dynamics theory, the kineto-elastic dynamic (KED) analysis of a mechanical system studies its vibration excited by the external load and inertial force, which suits for light-weight but high-speed working condition, as in Eq.(20); Meanwhile, the kineto-elastic static (KES) analysis considers the external load and inertial force as static force, and tries to solve the deformations of the system, which is more suitable for a heavy-load but low-speed case, as shown in Eq.(21).

$$
\begin{aligned}
& \mathbf{M} \mathscr{Q}^{\mathbb{Q}}+\mathbf{K} \boldsymbol{U}^{e}=\boldsymbol{F}-\mathbf{M} \mathscr{\mho}^{\mathbb{Q}} \\
& \mathbf{K} U^{e}=\boldsymbol{F}-\mathbf{M} \mathscr{\mho}^{\mathscr{e}}
\end{aligned}
$$

Since the TRICEPT manipulator discussed in this case is worked as a flexible forming machine, which satisfies the 2nd situation, therefore, KES analysis is suitablefor its model construction. Even so, a KED model is still constructed for convenience, with this model, KES analysis as well as vibration model, and other analyses could be deduced further on.

\subsubsection{Basic assumption and simplification}

The KED model is built based on the following assumptions:

a). The moving platform and the base are assumed to be rigid body, and all joints are considered 'ideal', which means no clearance and no deformation.

b). There is no coupling between rigid body displacement and elastic deformation of each linkage, so the real displacement of each coordinate can be calculated by a simple addition.

Using the standard spatial beam model in finite element method (FEM) theory, dynamics equation of each link can be formulated, then based on the 'deformation compatibility condition' and constraints, a dynamics model of the whole manipulator can be set up. Assuming each limb is consisted of two standard spatial beam elements, then the TRICEPT manipulator has overall 8 beam elements, as shown in Fig. 3. There are 2 elements in each actuated limb, where 3 nodes are set on 3 joints in each limb, and its 13 generalized coordinates are set based on joint constraints (4 in the U joint, 3 in the S joint and 6 in the actuated $\underline{\mathrm{P}}$ joint), as shown in Fig. 3(a); Accordingly, in the constrained limb, as in Fig. 3(b), there are 15 generalized coordinates in the limb (4 in the $U$ joint, 5 in the passive $P$ joint, and 6 in the fixed point $O_{b}$ ). (P.S: For the existence of actuated stiffness in each actuated limb, there exists elastic deformation in the moving direction of actuated $\underline{P}$ joint; on the contrary, $\mathrm{P}$ joint in the passive 
limb just moves as a follower, so there is no elastic deformation in that direction.)

\subsubsection{Dynamics model of each limb}

Based on the analysis above, the TRICEPT parallel manipulator is made up of 3 actuated limbs and 1 constrained limb. Since the 3actuated limbs are arranged symmetrically, also the constrained limb is constructed alike, only one of the actuated limbs will be taken as an example.

As shown in Fig. 4, each limb is made up of 2 linkages, each linkageis treated as a standard spatial beam element. Here only one linkage (element) in the actuated limb will be analyzed as an example.

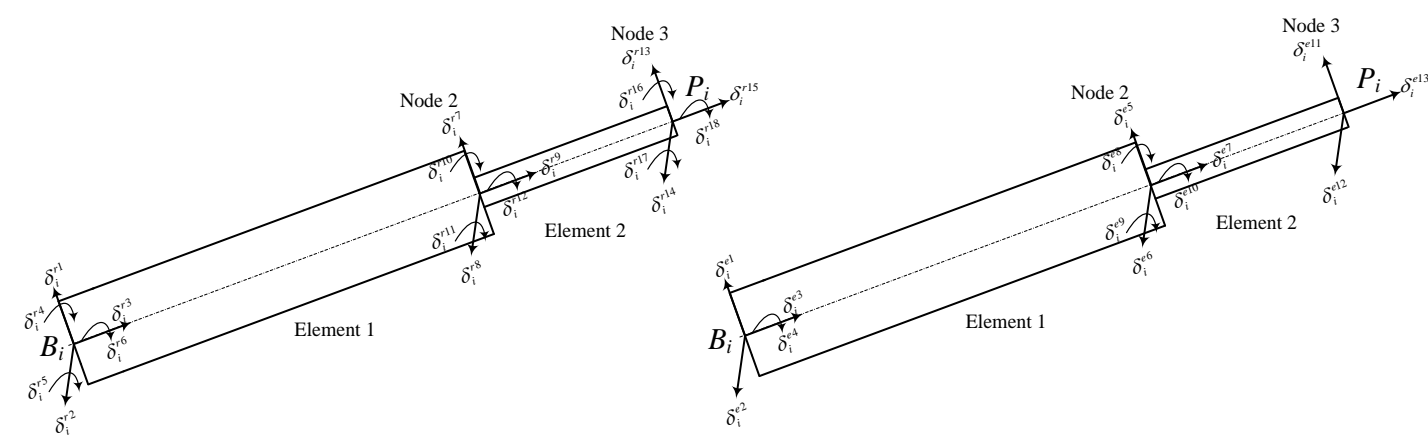

(a) Rigid-body displacement

(b)Elastic deformation

Figure4. Finite Element Model of the Actuated Limb with body-fixed coordinates

The elastic deformation on each node of the linkage is expressed in the body-fixed coordinate as $\delta_{i}^{e l}=\left[\begin{array}{lll}\delta_{i}^{e 1} & \cdots & \delta_{i}^{e 12}\end{array}\right]^{\mathrm{T}}, i=1 \sim 3$, where the elastic deformation on the direction of unconstrained degree of freedom(DOF) is zero; the rigid body displacement is $\delta_{i}^{r 1}=\left[\begin{array}{lll}\delta_{i}^{r 1} & \cdots & \delta_{i}^{r 12}\end{array}\right]^{\mathrm{T}}, i=1 \sim 3$, which can be calculated by rigid-body kinematics analysis. According to KED assumptions, the actual displacement of each node is considered a simple addition $\boldsymbol{\delta}_{i}^{1}=\boldsymbol{\delta}_{i}^{e 1}+\boldsymbol{\delta}_{i}^{\text {r1 }}$. So its kinetic energy and potential energy can be given by

$$
\begin{gathered}
T_{i}^{1}=\frac{1}{2}\left(\boldsymbol{\delta}_{i}^{\mathcal{1}_{1}}+\boldsymbol{\delta}_{i}^{\boldsymbol{\xi}^{1}}\right)^{\mathrm{T}} \mathbf{M}_{i}^{\delta 1}\left(\boldsymbol{\delta}_{i}^{\boldsymbol{\ell}_{1}}+\boldsymbol{\delta}_{i}^{\boldsymbol{k}_{1}^{1}}\right) \\
V_{i}^{1}=\frac{1}{2}\left(\boldsymbol{\delta}_{i}^{e 1}\right)^{T} \mathbf{K}_{i}^{\delta 1}\left(\boldsymbol{\delta}_{i}^{e 1}\right)
\end{gathered}
$$

where $\mathbf{M}_{i}^{\delta 1}, \mathbf{K}_{i}^{\text {\&1 }}$ denotes mass and stiffness matrix in the body-fixed coordinate of the linkage, their specific formulations can be found in any structural mechanics textbook.

Using Lagrange's second dynamics function, the dynamics differential equation of this linkage under its body-fixed coordinate system is given by

$$
\mathbf{M}_{i}^{\delta 1} \ddot{\boldsymbol{\delta}}_{i}^{e 1}+\mathbf{K}_{i}^{\delta 1} \boldsymbol{\delta}_{i}^{e 1}=\boldsymbol{f}_{i}^{\delta 1}+\boldsymbol{f}_{i}^{\text {ז̊1 }}-\mathbf{M}_{i}^{\delta 1} \ddot{\boldsymbol{\delta}}_{i}^{\mathrm{r} 1}
$$

Transforming to the reference coordinate frame $O_{b}-x_{b} y_{b} z_{b}$ yields

$$
\mathbf{M}_{i}^{1} \mathbf{m i t}_{i}^{1}+\mathbf{K}_{i}^{1} \mathbf{u}_{i}^{e 1}=\boldsymbol{f}_{i}^{1}+\boldsymbol{f}_{i}^{\mathrm{r1}}-\mathbf{M}_{i}^{1} \mathbf{u}_{i}^{\mathrm{r1}}
$$

where $\boldsymbol{u}_{i}^{e 1}=\mathbf{R}_{i}^{T} \boldsymbol{\xi}_{i}^{11}, \mathbf{M}_{i}^{1}=\mathbf{R}_{i} \mathbf{M}_{i}^{\delta 1} \mathbf{R}_{i}^{T}, \mathbf{K}_{i}^{1}=\mathbf{R}_{i} \mathbf{K}_{i}^{\delta 1} \mathbf{R}_{i}^{\mathrm{T}}, \boldsymbol{f}_{i}^{1}=\mathbf{R}_{i} \boldsymbol{f}_{i}^{\delta 1}, \boldsymbol{f}_{i}^{\mathrm{r1}}=\mathbf{R}_{i} \boldsymbol{f}_{i}^{\mathrm{r} 11}, \boldsymbol{f}_{i}^{\mathrm{r} 1}$ represents the force acting on the nodes between two elements, which is the generalized constraint force acting on the 
joint that can be solved by rigid-body dynamics analysis, $\boldsymbol{f}_{i}^{\delta 1}$ denotes the external force or torque acting on element $1, \mathbf{R}_{i}$ indicates the adjoint transformation matrix of body-fixed coordinate system $O_{i}-x_{i} y_{i} z_{i}$ with respect to reference coordinate frame $O_{b}-x_{b} y_{b} z_{b}, \mathbf{M}_{i}^{1}, \mathbf{K}_{i}^{1}$ denotes the mass and stiffness matrix in reference coordinate frame.

Dynamics modeling process of Element 2 in the actuated limb is similar, so in order to get the dynamics model of the limb, the two element models will be assembled into oneactuated limb model. According to the analysis before, the actuated limb has 13 generalized coordinates, with $\boldsymbol{U}_{i}^{e}=\left[\begin{array}{lll}U_{i}^{1} & \cdots & U_{i}^{13}\end{array}\right]^{T}, i=1 \sim 3$, being the expression in the reference coordinate $O_{b}-x_{b} y_{b} z_{b}$, as shown in Fig. 5(a).
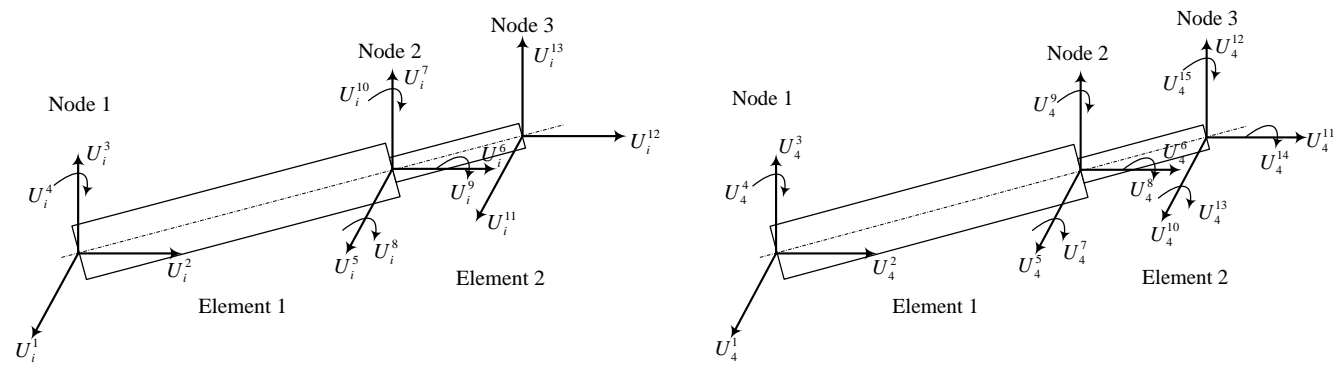

(a) (b)

Figure5.FEM of (a) Actuated Limb (b) Constrained Limb, with Generalized Coordinates in the reference coordinate

By using the Boolean assembling matrix

$$
\boldsymbol{u}_{i}^{e j}=\mathbf{B}_{i}^{e j} \boldsymbol{U}_{i}^{e}
$$

where $\mathbf{B}_{i}^{e}$ is the assembling matrix between the $j$-th element model and the $i$-th actuated limb model, their specific expressions are shown in the Appendix.

Then the actuated limb dynamics model in the reference coordinate $O_{b}-x_{b} y_{b} z_{b}$ is given by

$$
\mathbf{M}_{i} \dot{G}_{i}^{\mathbb{R}}+\mathbf{K}_{i} \boldsymbol{U}_{i}^{e}=\boldsymbol{F}_{i}+\boldsymbol{F}_{i}^{r}-\boldsymbol{F}_{i}^{*}
$$

where $\mathbf{M}_{i}, \mathbf{K}_{i}$ represents mass and stiffness matrix of the actuated limb, $\boldsymbol{F}_{i}$ denotes the external force acting on the limb, $F_{i}^{r}$ means the constrained force acting on the joints, $F_{i}^{*}$ is the inertial force, and their specific expression is given by

$$
\begin{aligned}
& \mathbf{M}_{i}=\sum_{j=1}^{2}\left(\mathbf{B}_{i}^{e j}\right)^{T} \mathbf{M}_{i}^{j}\left(\mathbf{B}_{i}^{e j}\right), \quad \mathbf{K}_{i}=\sum_{j=1}^{2}\left(\mathbf{B}_{i}^{e j}\right)^{T} \mathbf{K}_{i}^{j}\left(\mathbf{B}_{i}^{e j}\right), \quad \boldsymbol{F}_{i}=\sum_{j=1}^{2}\left(\mathbf{B}_{i}^{e j}\right)^{T} \boldsymbol{f}_{i}{ }^{j} \\
& \boldsymbol{F}_{i}^{r}=\sum_{j=1}^{2}\left(\mathbf{B}_{i}^{e j}\right)^{T} \boldsymbol{f}_{i}^{r j}, \boldsymbol{F}_{i}^{*}=\sum_{j=1}^{2}\left(\mathbf{B}_{i}^{e j}\right)^{T} \mathbf{M}_{i}^{j} \boldsymbol{G}_{i}^{\mathbf{Q}} .
\end{aligned}
$$

The modeling process of the constrained limb is similar, so in this section details will be omitted. Since there is no actuator in this limb, and one end of this limb is fixed with the platform, generalized coordinates here is different from the actuated limb, with $U_{4}^{e}=\left[\begin{array}{lll}U_{4}^{1} & \cdots & U_{4}^{15}\end{array}\right]^{T}$ being the expression in the reference coordinate. A similar dynamics formulation can be achieved like Eq.(27).

\subsubsection{Dynamics model of the whole manipulator}

Based on the analysis above, the overall generalized coordinates on every linkage of the manipulator 
$\operatorname{are} \boldsymbol{U}_{1}^{e(1 \times 13)}, \boldsymbol{U}_{2}^{e(1 \times 13)}, \boldsymbol{U}_{3}^{e(1 \times 13)}, \boldsymbol{U}_{4}^{e(1 \times 15)}$, but these coordinates are not independent, for all links are connected to the platform. Therefore, the deformation on the connecting joints (spherical joints on actuated limb or the fixed point on constrained limb) must have some 'deformation compatible' relations with the displacement of the platform. Since the moving platform is assumed to be rigid, here the generalized coordinates on the platform is defined as $\boldsymbol{U}_{0}^{\mathrm{r}}=\left[\begin{array}{lll}U_{0}^{1} & \cdots & U_{0}^{6}\end{array}\right]^{T}$. The nodes connecting each limb and the platform must follow the deformation compatibility condition. Since the translational displacements of the spherical joints in the actuated UPS limb and the platform is the same, which is given by

$$
\boldsymbol{U}_{i, 3}^{e}+\boldsymbol{U}_{i, 3}^{r}=\operatorname{Ad}_{g} \boldsymbol{U}_{0}^{r}=\left[\begin{array}{cc}
\mathbf{I}_{3 \times 3} & -\boldsymbol{\beta} \% \\
\mathbf{0}_{3 \times 3} & \mathbf{I}_{3 \times 3}
\end{array}\right] \boldsymbol{U}_{0}^{r}
$$

where $\boldsymbol{U}_{i, 3}^{e}=\left[\begin{array}{llllll}U_{i}^{11} & U_{i}^{12} & U_{i}^{13} & 0 & 0 & 0\end{array}\right]^{T}$ denotes the elastic deformation of the linkage point coincides with each spherical joint, $\boldsymbol{U}_{i, 3}^{r}$ is the corresponding rigid body displacement, $\operatorname{Ad}_{g}=\left[\begin{array}{ll}\mathbf{I}_{3 \times 3} & -8 \% \\ \mathbf{0}_{3 \times 3} & \mathbf{I}_{3 \times 3}\end{array}\right]$ means the adjoint transformation matrix, $\boldsymbol{r}^{i}$ represents the vector from $O_{p}$ to the center of spherical joint $P_{i}$, $i=1 \sim 3, \hat{\boldsymbol{r}}^{i}$ denotes the skew symmetric matrix of $\boldsymbol{r}^{i}, \mathbf{I}_{3 \times 3}$ is the $3 \times 3$ identity matrix.

Similarly, since the platform is assumed to be rigid, and the constrained limb is fixed on the platform at one end, based on the rigid body kinematics, the compatibility condition between platform and constrained UP limb can be formulated:

$$
\boldsymbol{U}_{4,3}^{e}+\boldsymbol{U}_{4,3}^{r}=\boldsymbol{U}_{0}^{r}
$$

where $\quad \boldsymbol{U}_{4,3}^{e}=\left[\begin{array}{llllll}U_{4}^{10} & U_{4}^{11} & U_{4}^{12} & U_{4}^{13} & U_{4}^{14} & U_{4}^{15}\end{array}\right]^{T}$ represents elastic deformation of the fixed point on the linkage; $\boldsymbol{U}_{4,3}^{r}$ is the corresponding rigid body displacement.

Based on above analysis, when the generalized coordinates on the platform $U_{0}^{\mathrm{r}}$ is considered, the three translational coordinates of the spherical joint node in the actuated U $\underline{P S}$ limb is eliminated, all six translational and rotational coordinates of the fixed point in the constrained limb is also removed. So the whole TRICEPT manipulator have 45 generalized coordinates in total:

$$
\boldsymbol{U}^{\boldsymbol{e}}=\left[\left(\boldsymbol{U}_{1}^{\boldsymbol{e}(1 \times 10)}\right)^{\mathrm{T}},\left(\boldsymbol{U}_{2}^{\boldsymbol{e}(1 \times 10)}\right)^{\mathrm{T}},\left(\boldsymbol{U}_{3}^{\boldsymbol{e}(1 \times 10)}\right)^{\mathrm{T}},\left(\boldsymbol{U}_{4}^{\boldsymbol{e}(1 \times 9)}\right)^{\mathrm{T}},\left(\boldsymbol{U}_{0}^{r(1 \times 6)}\right)^{\mathrm{T}}\right]^{\mathrm{T}}
$$

TheKED equation for the manipulator in the reference coordinate frame can be expressed as

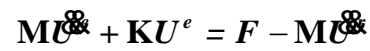

where $\mathbf{M}$, K represents overall mass and stiffness matrix, $\boldsymbol{F}$ denotes the external force acting on the manipulator, with

$$
\mathbf{M}=\sum_{i=1}^{4}\left(\mathbf{B}_{i}\right)^{T} \mathbf{M}_{i}\left(\mathbf{B}_{i}\right), \quad \boldsymbol{F}=\sum_{i=0}^{4}\left(\mathbf{B}_{i}\right)^{\mathrm{T}} \boldsymbol{F}_{i}, \quad \mathbf{K}=\sum_{\mathrm{i}=1}^{4}\left(\mathbf{B}_{i}\right)^{T} \mathbf{K}_{i}\left(\mathbf{B}_{i}\right) .
$$

where $\mathbf{B}_{i}$ is generalized coordinates assembling matrix between the $i$-th limb and the whole manipulator, their specific expressions are shown in the Appendix. 
Then KES model can be acquired from Eq.(31), in the form

$$
\mathbf{K} \boldsymbol{U}^{e}=\boldsymbol{F}-\mathbf{M} \mathbb{G}^{\mathbb{k}}
$$

\subsubsection{End-effector stiffness model}

According to the analysis in Section 2.2.2, the reduced stiffness model is used in our study. By comparing Eq.(7) and Eq.(32), the preserved generalized coordinates $\boldsymbol{U}^{p}$ in the TRICEPT dynamics model is $\boldsymbol{U}_{0}^{r(1 \times 6)}$ in Eq.(30), then the $45 \times 45$ stiffness matrix Kcan be divided accordingly, then the KES model (32) can be divided into

$$
\left(\begin{array}{ll}
\mathbf{K}_{\mathrm{cc}} & \mathbf{K}_{\mathrm{cp}} \\
\mathbf{K}_{\mathrm{cp}}^{\mathrm{T}} & \mathbf{K}_{\mathrm{pp}}
\end{array}\right)\left(\begin{array}{l}
\boldsymbol{U}^{c} \\
\boldsymbol{U}^{p}
\end{array}\right)=\left(\begin{array}{l}
\boldsymbol{F}^{c} \\
\boldsymbol{F}^{p}
\end{array}\right)
$$

where $\mathbf{K}=\left(\begin{array}{ll}\mathbf{K}_{\mathrm{cc}} & \mathbf{K}_{\mathrm{cp}} \\ \mathbf{K}_{\mathrm{cp}}^{\mathrm{T}} & \mathbf{K}_{\mathrm{pp}}\end{array}\right), \boldsymbol{F}-\mathbf{M} \ddot{\boldsymbol{U}}^{r}=\left(\begin{array}{l}\boldsymbol{F}^{c} \\ \boldsymbol{F}^{p}\end{array}\right)$.

With this division and Guyan's static condensation method in Eq.(10), the $6 \times 6$ end-effector stiffness model of TRICEPT parallel manipulator is derived accordingly

$$
\mathbf{K}_{\text {end }}=\mathbf{K}_{\mathrm{pp}}-\mathbf{K}_{\mathrm{cp}}^{\mathrm{T}} \mathbf{K}_{\mathrm{cc}}^{\mathrm{T}} \mathbf{K}_{\mathrm{cp}}
$$

\subsection{Optimization model of the TRICEPT manipulator}

In our study, the TRICEPT parallel manipulator is assumed to function as a flexible forming machine, where the manipulator undertakes heavy load from the plastic deformation of metal plates, while the machining speed is slow. In view of the actual machining situations in our lab, the path of the tool head is defined as a spiral curve with spherical coordinates radius of $0.05 \mathrm{~m}$ and amount of feed with $0.001 \mathrm{~m}$.

$$
\left\{\begin{array}{l}
x=0.05 \sin \left(\frac{\pi t}{18}-\frac{\pi}{36}\right) \\
y=0.05 \cos \left(\frac{\pi t}{18}-\frac{\pi}{36}\right) \\
z=0.9+0.001 t
\end{array}\right.
$$

where t represents time, and the path is defined in the reference coordinate frame.

The CAD model and the working path is shown in Fig.6.

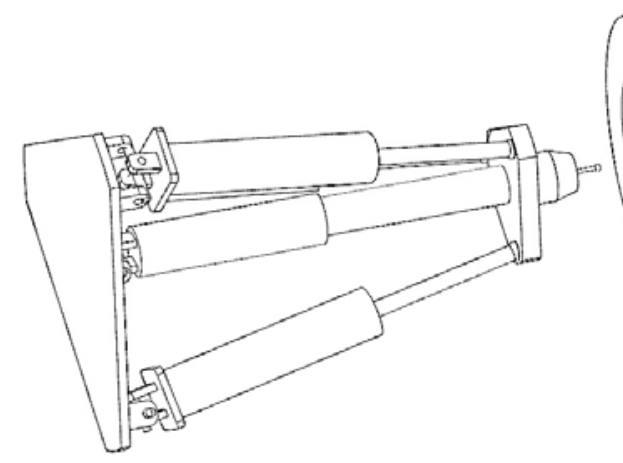

a) CAD model of the machined part

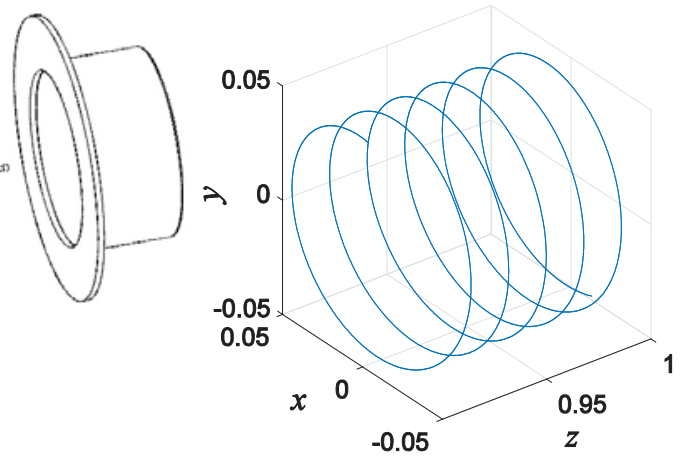

b) machining path

Figure 6. Diagram of the machining process

Based on experimental results, the force acting on the end-effector (in unit $\mathrm{N}$ ) due to the plate deformation is 


$$
\left\{\begin{array}{l}
F_{x}=3000 \sin (20 \pi t) \\
F_{y}=2000 \cos (20 \pi t) \\
F_{z}=2000|\sin (20 \pi t)|
\end{array}\right.
$$

\subsubsection{Design variables of the TRICEPT manipulator}

Based on the working condition defined above and dynamics introduced in 4.1, the parameter optimization model can be constructed. The thickness of the moving platform is $h=0.01 \mathrm{~m}$, and also the diameter of circumscribed circle of the base is set as a fixed value $d_{b}=0.45 \mathrm{~m}$ in view of the actual working environment, so the ratio $\alpha_{p}$ between the diameter of circumscribed circle of the moving platform and the base is one of the optimization design variable; Since 3 actuated limbs of the TRICEPT manipulator are symmetrical, and the lower linkage of each limb is connected with actuator, so the length of the lower linkage of each actuated limb $l_{a}$ is also a design variable, so is the length of the lower linkage of the constrained limb $l_{c}$. Since dynamic performances are the focus of this study as explained in Section 3.2, diameter of each linkage should also be design variables, where $d_{a}$ is the cross-sectional diameter of lower linkage of the actuated limb, and $d_{c}$ corresponds to the constrained limb, with $\alpha_{a}, \alpha_{c}$ being the ratio between the cross-sectional diameter of upper linkage and lower linkage in each limb.

In summary, the overall optimization design variables for the TRICEPT manipulator include:

$$
\boldsymbol{X}=\left[l_{a}, l_{c}, d_{a}, d_{c}, \alpha_{a}, \alpha_{c}, \alpha_{p}\right]
$$

where $\boldsymbol{X}_{d}=\left[l_{a}, l_{c}\right]$ are dimensional variables, and $\boldsymbol{X}_{s}=\left[d_{a}, d_{c}, \alpha_{a}, \alpha_{c}, \alpha_{p}\right]$ denote structural variables, and bounds of these variables are :

$$
l_{a}, l_{c} \in[0.3,0.7] m, \quad d_{a}, d_{c} \in[0.05,0.13] m, \quad \alpha_{a}, \alpha_{c} \in[0.4,0.9], \quad \alpha_{p} \in[0.4,0.8] .
$$

Constraints of these design variable should involve the ratio between the biggest and the smallest value of each kind of parameter.

1) Linkage length ratio: $C_{1}=L_{\text {Ratio }}=l_{\max } / l_{\min } \leq 3$;

2) Linkage diameter ratio: $C_{2}=d_{\text {Ratio }}=d_{\text {max }} / d_{\text {min }} \leq 3$;

\subsubsection{Optimization model construction}

From the optimization evaluation indices introduced in Section 3.2, all four dynamic optimization objectives are considered in this TRICEPT manipulator case, two constraints are involved as in Section 4.2.1, then the optimization model of the manipulator can be formulated

$$
\left\{\begin{array}{l}
\min \quad F=-\omega_{1} \varphi_{\mathrm{SDD}}\left(\boldsymbol{X}_{d}, \boldsymbol{X}_{s}\right)+\omega_{2} \frac{1}{\varphi_{\mathrm{SEE}}^{a}\left(\boldsymbol{X}_{d}, \boldsymbol{X}_{s}\right)}+\omega_{3} \frac{1}{\varphi_{\mathrm{SEE}}^{m}\left(\boldsymbol{X}_{d}, \boldsymbol{X}_{s}\right)}+\omega_{4} \varphi_{M}\left(\boldsymbol{X}_{d}, \boldsymbol{X}_{s}\right) \\
\text { subject to: } f_{\text {constraint }}=\sum_{i=1}^{n} w_{i} C_{i}=0 \quad(i=1,2)
\end{array}\right.
$$

In this paper, different performances are taken into account in the proposed optimization problem, the multi-objective function is just a compromise of several performance indices, rather than a concrete evaluation of a particular one. Therefore, the unit inconsistence between the multiple objectives is not 
taken into consideration in our model. Besides, in this paper, all four optimization objectives are equally important to the design requirement, so the weighted parameters are set to be the same.

\subsubsection{Setup for the particle swarm optimization (PSO) algorithm}

Since the number of design variables are 7, the particle dimension is set to 7 accordingly; the population of swarm size is set to be 100; and the maximum number of consecutive iteration steps within permissible error range $10^{-6}$ is 30 .

\subsection{Optimization results}

Programming the aforementioned optimization model using Matlab software, the optimum results are listed in Table 2; then the dimension and structure parameters can be deduced accordingly.

Table 2. Results of the optimization

\begin{tabular}{|c|c|c|c|}
\hline $\begin{array}{c}\text { Design } \\
\text { Variable }\end{array}$ & $\begin{array}{c}\text { Before } \\
\text { Optimization }\end{array}$ & $\begin{array}{c}\text { After } \\
\text { Optimization }\end{array}$ & Deduced Parameter \\
\hline$l_{a}$ & $0.4 m$ & $0.3 m$ & -- \\
\hline$l_{c}$ & $0.45 \mathrm{~m}$ & $0.63389 \mathrm{~m}$ & -- \\
\hline$d_{a}$ & $0.05 \mathrm{~m}$ & $0.1214 m$ & -- \\
\hline$d_{c}$ & $0.075 \mathrm{~m}$ & $0.11541 \mathrm{~m}$ & - \\
\hline$\alpha_{a}$ & 0.5 & 0.80441 & $\begin{array}{l}\text { Cross-sectional diameter of the upper linkage of actuated limb: } \\
\qquad d_{a} \alpha_{a}=0.0976 \mathrm{~m}\end{array}$ \\
\hline$\alpha_{c}$ & 0.67 & 0.9 & $\begin{array}{l}\text { Cross-sectional diameter of the upper linkage of constrained limb: } \\
\qquad d_{c} \alpha_{c}=0.104 \mathrm{~m}\end{array}$ \\
\hline$\alpha_{p}$ & 0.44 & 0.4 & $\begin{array}{l}\text { Diameter of the circumcircle of the moving platform: } d_{b} \alpha_{p}= \\
\qquad 0.18 \mathrm{~m}\end{array}$ \\
\hline
\end{tabular}

The results of objective function before and after the optimization, by using the original and optimized value of design variables respectively, is listed in Table 3.

Table 3. Value of objective function before and after optimization

\begin{tabular}{ccc}
\hline \multicolumn{2}{c}{ Table 3. Value of objective function before and after optimization } \\
\hline$F$ & 3.3494 & After optimization \\
$\varphi_{\mathrm{SDD}}$ & 0.9931 & 2.3443 \\
$1 / \varphi_{\mathrm{SEE}}^{a}$ & $1.2786 \mathrm{e}-10$ & 0.3371 \\
$1 / \varphi_{\mathrm{SEE}}^{m}$ & $1.2796 \mathrm{e}-10$ & $2.2983 \mathrm{e}-11$ \\
$\varphi_{M}$ & 2.3499 & $2.2991 \mathrm{e}-11$ \\
\hline \hline
\end{tabular}

From the results, it can be easily seen that after optimization, fitness value ${ }^{3}$ is reduced from 3.34 to 2.3455, and each of the three individual performance indices has been improved. So this parameter optimization process could indeed enhance the dynamic performances: the stiffness of each elastic part distributed in the system more evenly which is concerned most since its heavy-load working condition; the Cartesian stiffness of the end-effector is enhanced; and of course the weight of the whole manipulator is also dropped.

\subsection{Results analysis}

To have a better understanding of our optimization method, the stiffness distribution of each individual linkage in the manipulator, as well as end-effector stiffness along the working path before

3 'Fitness value' is a commonly used phrase in optimization algorithm, which means value of the objective function. 
and after optimization is illustrated in the following.

\subsubsection{Stiffness distribution of each linkage}

Following the definition in Eq.(4), the stiffness density distribution $\gamma_{i}^{\rho}$ of the $i$-th linkage in the whole manipulator along the designated working path(35) before and after optimization can be illustrated, as shown in Fig.7 and 8. Also, the corresponding comparison is listed in Table 4.

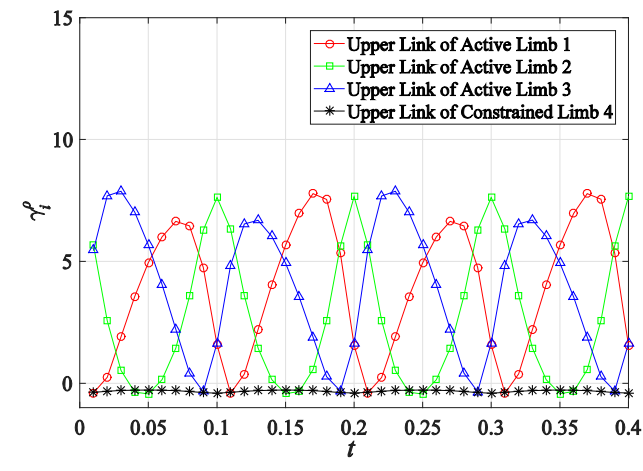

a). Upper Link in Each Limb.

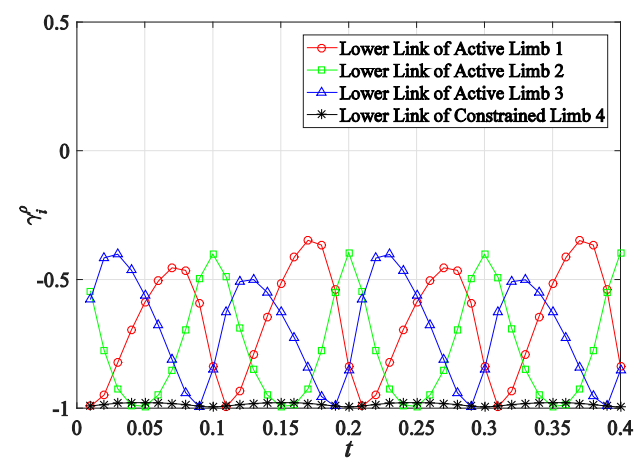

b). Lower Link in Each Limb

Figure7.Stiffness distribution $\gamma_{i}^{\rho}$ of each linkage along the designated path (Before optimization)

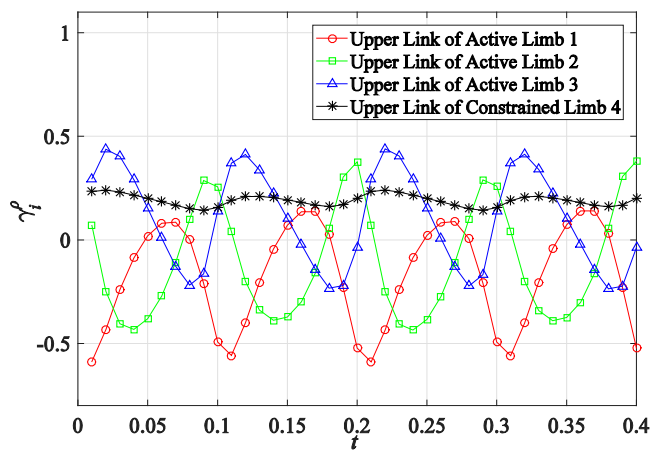

a). Upper Link in Each Limb.

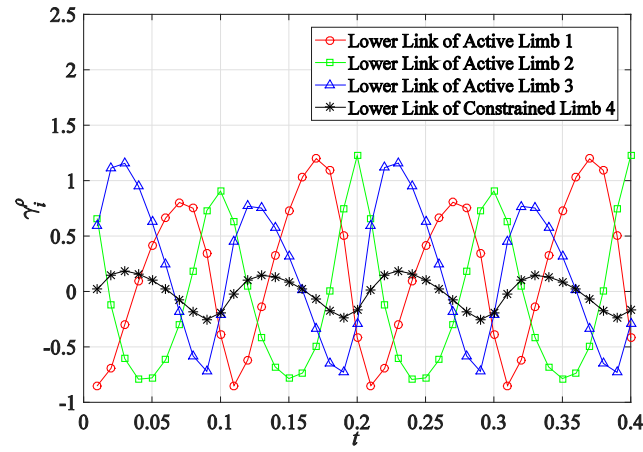

b). Lower Link in Each Limb

Figure8.Stiffness distribution $\gamma_{i}^{\rho}$ of each linkage along the designated path (Afteroptimization)

Table 4. stiffness distribution comparison before/after optimization

\begin{tabular}{ccc}
\hline \hline Stiffness distribution $\gamma_{i}^{\rho}$ & Before Optimization & After Optimization \\
\hline Upper Links in Actuated Limbs & $0.8 \sim 8$ & $-0.6 \sim 0.5$ \\
Lower Links in Actuated Limbs & $-0.9 \sim-0.7$ & $-0.8 \sim 1.2$ \\
Upper Links in Constrained Limb & $-0.6 \sim-0.5$ & $0.1 \sim 0.3$ \\
Lower Links in Constrained Limb & $-0.9 \sim-0.8$ & $-0.3 \sim-0.2$ \\
\hline \hline
\end{tabular}

From the results, the stiffness distribution $\gamma_{i}^{\rho}$ of the upper linkage in actuated limbs is around $0.8 \sim 8$, and the peak value 8 indicates that the elastic deformation energy density of this part is 8 times bigger than average in that situation, which is not allowed in heavy-load working environment since it is the most vulnerable part and could possibly put the whole system 'in danger'. After optimization, stiffness distribution $\gamma_{i}^{\rho}$ of almost all parts in the manipulator is in the range $-0.8 \sim 1.2$, which based 
on the definition in Eq.(4), is a tremendous improvement in stiffness distribution for the whole system.

\subsubsection{End-effector stiffness comparison}

Using the model condensation method introduced in Section 3.2.2, the end-effector stiffness index $k_{e}$ can be calculated using parameters before and after optimization, the corresponding results are shown in Fig. 9. Since the TRICEPT manipulator is working on a path that is just a small region in the workspace, the end-effector stiffness is also varying in a subtle way, as shown in Fig. 8.

It can be seen that the average end-effector stiffness is greatly improved (about 6 times) after the optimization process, which is obvious since the optimized parameters are greater than the original one to some extent.

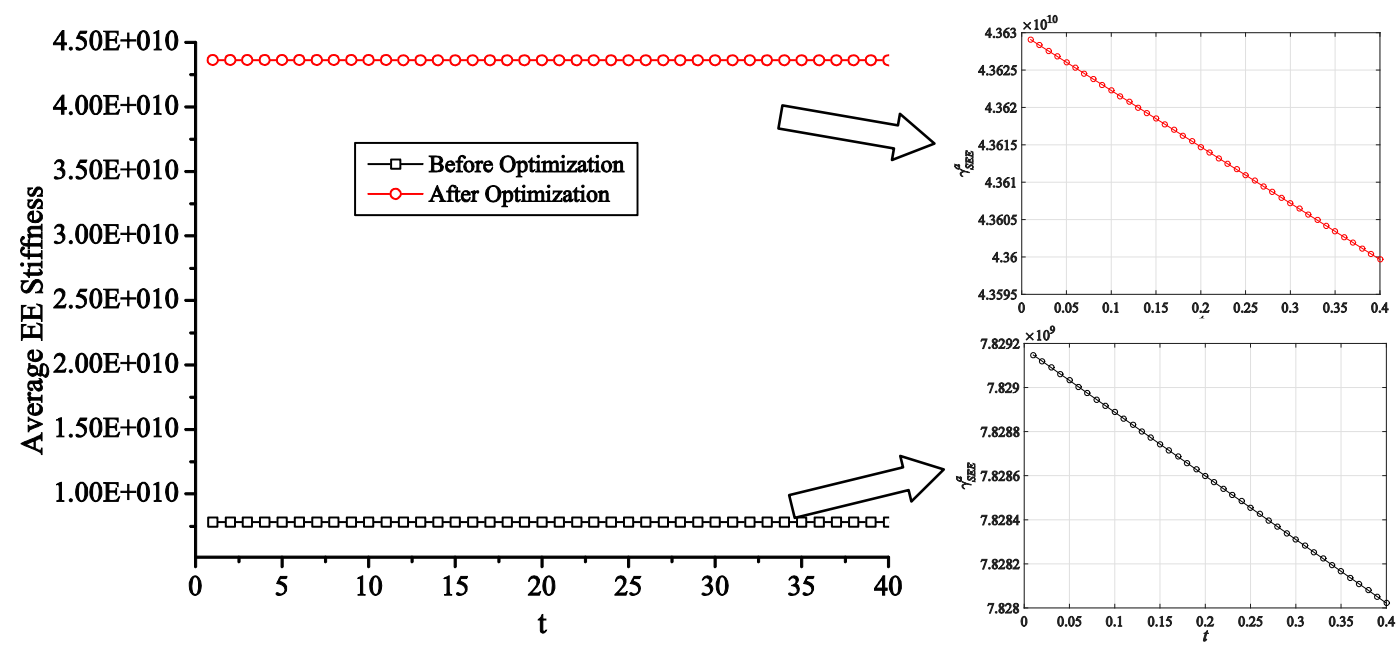

Figure 9. Average End-effector stiffness before/after optimization

In the meantime, from Table 3, the minimum end-effector stiffness $\varphi_{\mathrm{SEE}}^{m}$ is also greatly improved, which means that after optimization, this manipulator can better avoid the worst stiffness case along the practical working path.

\section{Conclusion}

Targeting at the specific heavy-load working condition of parallel manipulators, unlike traditional kinematic design as well as dynamic stiffness design which focuses on end-effector stiffness performance, this paper provides a new stiffness distribution evaluation index, which puts emphasis on the stiffness distribution among different elastic parts in the entire manipulator.

By adopting this stiffness distribution index, together with three other dynamic performance indices and some kinematic constraints, a multi-objective optimization model can be constructed, and the corresponding optimum parameter can be obtained by applying PSO algorithm.

A case study of the widely used industrial robot TRICEPT is introduced to illustrate the effectiveness of the defined index and the superiority of optimization process. Based on the results before and after optimization, it is obviously that the dynamic performances can be improved tremendously.

\section{Acknowledgement}

This research work is partially supported by grants from the National Science Foundation of China (NSFC) under Grant No.51305256 and No.51605300, the National Basic Research Program of China (Grant No.2014CB046600). The authors are grateful for these financial supports.

\section{Appendix}

1.The assembling matrix between the $j$-th element model and the $i$-th actuated limb model: 

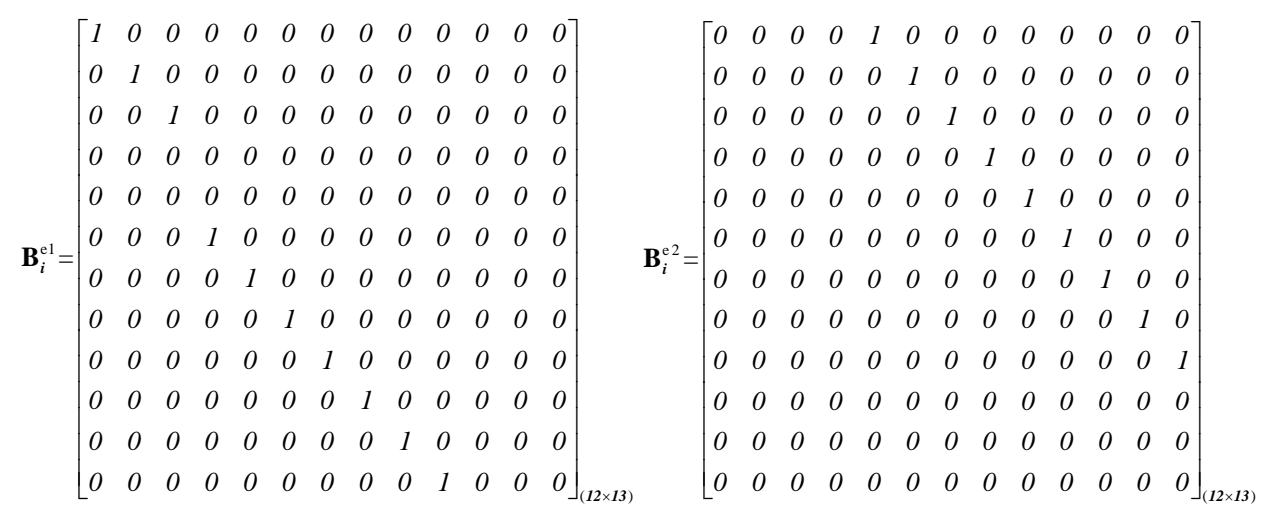

The assembling matrix between the element model and the constrained limb model:
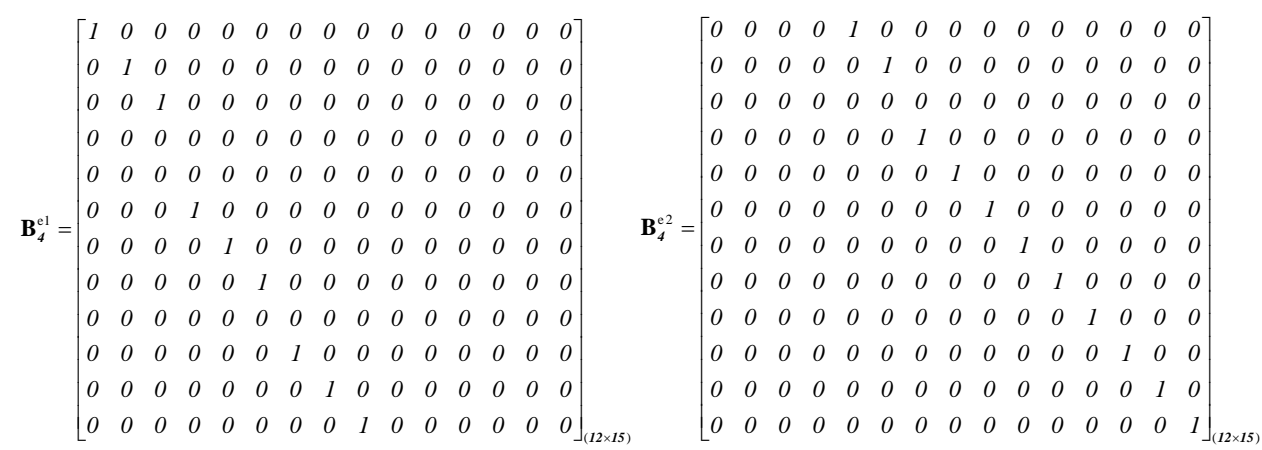

The assembling matrix between the $i$-th limb and the whole manipulator

$$
\begin{aligned}
& \mathbf{B}_{1}=\left[\begin{array}{cccccc}
\mathbf{I}_{10 \times 10} & \mathbf{0}_{10 \times 10} & \mathbf{0}_{10 \times 10} & \mathbf{0}_{10 \times 9} & \mathbf{0}_{10 \times 3} & \mathbf{0}_{10 \times 3} \\
\mathbf{0}_{3 \times 10} & \mathbf{0}_{3 \times 10} & \mathbf{0}_{3 \times 10} & \mathbf{0}_{3 \times 9} & \mathbf{I}_{3 \times 3} & -\hat{\boldsymbol{r}}_{1}
\end{array}\right]_{(13 \times 45)} \\
& \mathbf{B}_{2}=\left[\begin{array}{cccccc}
\mathbf{0}_{10 \times 10} & \mathbf{I}_{10 \times 10} & \mathbf{0}_{10 \times 10} & \mathbf{0}_{10 \times 9} & \mathbf{0}_{10 \times 3} & \mathbf{0}_{10 \times 3} \\
\mathbf{0}_{3 \times 10} & \mathbf{0}_{3 \times 10} & \mathbf{0}_{3 \times 10} & \mathbf{0}_{3 \times 9} & \mathbf{I}_{3 \times 3} & -\hat{\boldsymbol{r}}_{2}
\end{array}\right]_{(13 \times 45)} \\
& \mathbf{B}_{3}=\left[\begin{array}{llllll}
\mathbf{0}_{10 \times 10} & \mathbf{0}_{10 \times 10} & \mathbf{I}_{10 \times 10} & \mathbf{0}_{10 \times 9} & \mathbf{0}_{10 \times 3} & \mathbf{0}_{10 \times 3} \\
\mathbf{0}_{3 \times 10} & \mathbf{0}_{3 \times 10} & \mathbf{0}_{3 \times 10} & \mathbf{0}_{3 \times 9} & \mathbf{I}_{3 \times 3} & -\hat{\boldsymbol{r}}_{3}
\end{array}\right]_{(13 \times 45)} \\
& \mathbf{B}_{4}=\left[\begin{array}{llllll}
\mathbf{0}_{9 \times 9} & \mathbf{0}_{9 \times 9} & \mathbf{0}_{9 \times 9} & \mathbf{I}_{9 \times 9} & \mathbf{0}_{9 \times 3} & \mathbf{0}_{9 \times 3} \\
\mathbf{0}_{3 \times 9} & \mathbf{0}_{3 \times 9} & \mathbf{0}_{3 \times 9} & \mathbf{0}_{3 \times 9} & \mathbf{I}_{3 \times 3} & -\hat{\boldsymbol{r}}_{4} \\
\mathbf{0}_{3 \times 9} & \mathbf{0}_{3 \times 9} & \mathbf{0}_{3 \times 9} & \mathbf{0}_{3 \times 9} & \mathbf{0}_{3 \times 3} & \mathbf{I}_{3 \times 3}
\end{array}\right]_{(15 \times 45)}
\end{aligned}
$$

\section{Reference}

[1] J.P. Merlet, Parallel robots, Springer Science \& Business Media, 2012.

[2] J. Kim, C. Park, J. Kim, F.C. Park, Performance analysis of parallel mechanism architectures for CNC machining applications, Journal of Manufacturing Science and Engineering, 122 (1999) 753-759.

[3] M.A. Hosseini, H.M. Daniali, Cartesian workspace optimization of Tricept parallel manipulator with machining application, Robotica, 33 (2015) 1948-1957.

[4] J.K. Salisbury, J.J. Craig, Articulated hands: force control and kinematic issues, The International Journal of Robotics Research, 1 (1982) 4-17.

[5] T. Huang, Z. Li, M. Li, D.G. Chetwynd, C.M. Gosselin, Conceptual design and dimensional synthesis of a novel 2-DOF 
translational parallel robot for pick-and-place operations, Journal of Mechanical Design, Transactions of the ASME, 126 (2004) 449-455.

[6] C. Gosselin, J. Angeles, Optimum kinematic design of a planar three-degree-of-freedom parallel manipulator, Journal of mechanisms, transmissions, and automation in design, 110 (1988) 35-41.

[7] O. Altuzarra, C. Pinto, B. Sandru, A. Hernandez, Optimal dimensioning for parallel manipulators: Workspace, dexterity, and energy, Journal of Mechanical Design, Transactions of the ASME, 133 (2011).

[8] A.B.K. Rao, P.V.M. Rao, S.K. Saha, Dimensional design of Hexaslides for optimal workspace and dexterity, IEEE Transactions on Robotics, 21 (2005) 444-449.

[9] C.M. Gosselin, Dexterity indices for planar and spatial robotic manipulators, 1990, pp. 650-655.

[10] T. Yoshikawa, Manipulability of robotic mechanisms, International Journal of Robotics Research, 4 (1985) 3-9.

[11] J. Wang, C. Wu, X.J. Liu, Performance evaluation of parallel manipulators: motion/force transmissibility and its index, Mechanism and Machine Theory, 45 (2010) 1462-1476.

[12] L. Zhang, J. Mei, X. Zhao, T. Huang, Dimensional synthesis of the Delta robot using transmission angle constraints, Robotica, 30 (2012) 343-349.

[13] V.T. Portman, V.S. Chapsky, Y. Shneor, Evaluation and optimization of dynamic stiffness values of the PKMs: Collinear stiffness value approach, Mechanism and Machine Theory, 74 (2014) 216-244.

[14] S. Jin, J. Kim, T. Seo, Optimization of a redundantly actuated 5R symmetrical parallel mechanism based on structural stiffness, Robotica, 33 (2015) 1973-1983.

[15] S. Briot, A. Pashkevich, D. Chablat, On the optimal design of parallel robots taking into account their deformations and natural frequencies, ASME 2009 International Design Engineering Technical Conferences and Computers and Information in Engineering Conference, American Society of Mechanical Engineers, 2009, pp. 367-376.

[16] J. Ryu, J. Cha, Volumetric error analysis and architecture optimization for accuracy of HexaSlide type parallel manipulators, Mechanism and Machine Theory, 38 (2003) 227-240.

[17] S. Kucuk, Energy minimization for 3-RRR fully planar parallel manipulator using particle swarm optimization, Mechanism and Machine Theory, 62 (2013) 129-149.

[18] Y. Zhao, Dynamic optimum design of a three translational degrees of freedom parallel robot while considering anisotropic property, Robotics and Computer-Integrated Manufacturing, (2012).

[19] S. Liu, T. Huang, J. Mei, X. Zhao, P. Wang, D.G. Chetwynd, Optimal design of a 4-DOF SCARA type parallel robot using dynamic performance indices and angular constraints, Journal of Mechanisms and Robotics, 4 (2012) 031005.

[20] T. Huang, S. Liu, J. Mei, D.G. Chetwynd, Optimal design of a 2-DOF pick-and-place parallel robot using dynamic performance indices and angular constraints, Mechanism and Machine Theory, 70 (2013) 246-253.

[21] K. Deb, Multi-objective optimization using evolutionary algorithms, John Wiley \& Sons, 2001.

[22] Y. Li, Y. Ma, S. Liu, Z. Luo, J. Mei, T. Huang, D. Chetwynd, Integrated design of a 4-DOF high-speed pick-and-place parallel robot, CIRP Annals-Manufacturing Technology, 63 (2014) 185-188.

[23] R. Kelaiaia, O. Company, A. Zaatri, Multiobjective optimization of a linear Delta parallel robot, Mechanism and Machine Theory, 50 (2012) 159-178.

[24] B. Bounab, Multi-objective optimal design based kineto-elastostatic performance for the DELTA parallel mechanism, Robotica, 34 (2016) 258-273.

[25] S.D. Stan, R. Bălan, V. Mătiesạ, Multi-objective design optimization of mini parallel robots using genetic algorithms, IEEE International Symposium on Industrial Electronics, 2007, pp. 2173-2178.

[26] F. Hao, J.P. Merlet, Multi-criteria optimal design of parallel manipulators based on interval analysis, Mechanism and Machine Theory, 40 (2005) 157-171.

[27] O. Altuzarra, A. Hernandez, O. Salgado, J. Angeles, Multiobjective optimum design of a symmetric parallel schönfliesmotion generator, Journal of Mechanical Design, Transactions of the ASME, 131 (2009) 0310021-03100211. 
[28] R. Kelaiaia, A. Zaatri, L. Chikh, Some investigations into the optimal dimensional synthesis of parallel robots, The International Journal of Advanced Manufacturing Technology, (2015) 1-14.

[29] H. Bruyninckx, J.D. Schutter, Specification of force-controlled actions in the "task frame formalism”-a synthesis, Robotics and Automation, IEEE Transactions on, 12 (1996) 581-589.

[30] J. Duffy, Statics and kinematics with applications to robotics, Cambridge University Press, 2007.

[31] G. Alici, B. Shirinzadeh, Enhanced stiffness modeling, identification and characterization for robot manipulators, Robotics, IEEE Transactions on, 21 (2005) 554-564.

[32] F. Xi, D. Zhang, C.M. Mechefske, S.Y. Lang, Global kinetostatic modelling of tripod-based parallel kinematic machine, Mechanism and Machine Theory, 39 (2004) 357-377.

[33] C. Gosselin, Stiffness mapping for parallel manipulators, Robotics and Automation, IEEE Transactions on, 6 (1990) 377-382.

[34] L.W. Tsai, Robot analysis: the mechanics of serial and parallel manipulators, John Wiley \& Sons, 1999.

[35] G. Carbone, Stiffness evaluation of multibody robotic systems, PhD Dissertation, LARM, University of Cassino, Cassino, 2003.

[36] J. Lončarić, Normal forms of stiffness and compliance matrices, Robotics and Automation, IEEE Journal of, 3 (1987) 567-572.

[37] Q. Xu, Y. Li, GA-based architecture optimization of a 3-PUU parallel manipulator for stiffness performance, Intelligent Control and Automation, 2006. WCICA 2006. The Sixth World Congress on, IEEE, 2006, pp. 9099-9103.

[38] E. Courteille, D. Deblaise, P. Maurine, Design optimization of a delta-like parallel robot through global stiffness performance evaluation, Intelligent Robots and Systems, 2009. IROS 2009. IEEE/RSJ International Conference on, IEEE, 2009, pp. 5159-5166.

[39] G. Carbone, M. Ceccarelli, Comparison of indices for stiffness performance evaluation, Frontiers of Mechanical Engineering in China, 5 (2010) 270-278.

[40] G. Carbone, M. Ceccarelli, A procedure for experimental evaluation of Cartesian stiffness matrix, 15th CISM-IFToMM Symposium on Robot Design, Dynamics and Control Romansy Montreal, CD Proceedings, 2004, pp. 4-24.

[41] G. Chen, H. Wang, Z. Lin, X. Lai, The principal axes decomposition of spatial stiffness matrices, IEEE Transactions on Robotics, 31 (2015) 191-207.

[42] X.J. Liu, Z.L. Jin, F. Gao, Optimum design of 3-DOF spherical parallel manipulators with respect to the conditioning and stiffness indices, Mechanism and Machine Theory, 35 (2000) 1257-1267.

[43] H.S. Kim, L.W. Tsai, Design optimization of a Cartesian parallel manipulator, Journal of Mechanical Design, 125 (2003) 43-51.

[44] S. Yan, S. Ong, A. Nee, Stiffness analysis of parallelogram-type parallel manipulators using a strain energy method, Robotics and Computer-Integrated Manufacturing, 37 (2016) 13-22.

[45] T. Huang, X. Zhao, D.J. Whitehouse, Stiffness estimation of a tripod-based parallel kinematic machine, Robotics and Automation, IEEE Transactions on, 18 (2002) 50-58.

[46] C.M. Gosselin, D. Zhang, Stiffness analysis of parallel mechanisms using a lumped model, International Journal of Robotics and Automation, 17 (2002) 17-27.

[47] J. Loncaric, Geometrical analysis of compliant mechanisms in robotics (euclidean group, elastic systems, generalized springs, (1985).

[48] O.C. Zienkiewicz, R.L. Taylor, The finite element method: solid mechanics, Butterworth-heinemann, 2000.

[49] H. Li, Z. Yang, T. Huang, Dynamics and elasto-dynamics optimization of a 2-DOF planar parallel pick-and-place robot with flexible links, Structural and Multidisciplinary Optimization, 38 (2009) 195-204.

[50] L. Zhang, Y. Song, Optimal design of the Delta robot based on dynamics, Robotics and Automation (ICRA), 2011 IEEE International Conference on, IEEE, 2011, pp. 336-341. 
[51] R. Guyan, Reduction of mass and stiffness matrices, AIAA journal, 3 (1965) 380.

[52] M. Ruggiu, Cartesian stiffness matrix mapping of a translational parallel mechanism with elastic joints, International Journal of Advanced Robotic Systems, 9 (2012).

[53] J. Kennedy, Particle Swarm Optimization, in: C. Sammut, G.I. Webb (Eds.) Encyclopedia of machine learning, Springer US, Boston, MA, 1998, pp. 760-766.

[54] B.Y. Shi, R. Eberhart, A modified particle swarm optimizer, Evolutionary Computation Proceedings, IEEE International Conference on, 1998, pp. 69-73.

[55] M. Clerc, J. Kennedy, The particle swarm - explosion, stability, and convergence in a multidimensional complex space, IEEE Transactions on Evolutionary Computation, 6 (2002) 58-73.

[56] S. Joshi, L.W. Tsai, A comparison study of two 3-DOF parallel manipulators: One with three and the other with four supporting legs, IEEE Transactions on Robotics and Automation, 19 (2003) 200-209.

[57] M. Li, T. Huang, J. Mei, X. Zhao, D.G. Chetwynd, S.J. Hu, Dynamic formulation and performance comparison of the 3-DOF modules of two reconfigurable PKM - The Tricept and the Trivariant, Journal of Mechanical Design, Transactions of the ASME, 127 (2005) 1129-1136. 

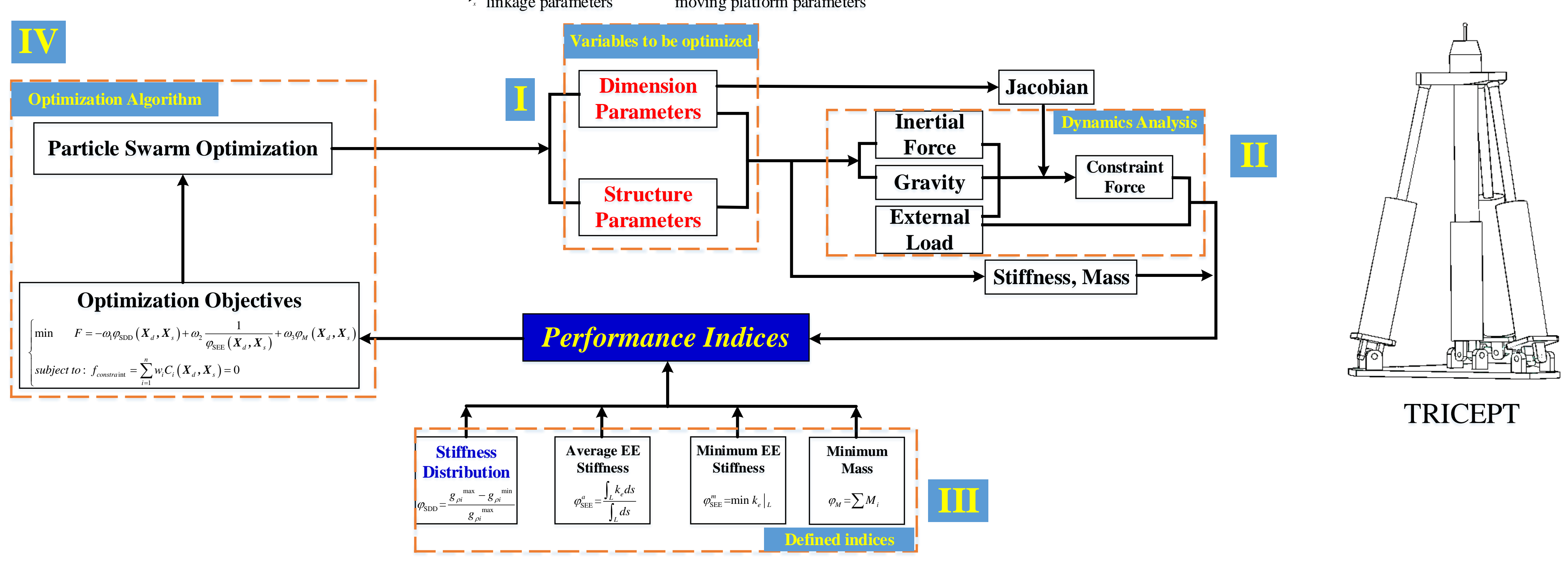